\title{
Improvement of cholesterol levels and reduction of cardiovascular risk via the consumption of phytosterols
}

\author{
Rosa M. Ortega ${ }^{1}$, Ana Palencia ${ }^{2}$ and Ana M López-Sobaler ${ }^{1}$ \\ ${ }^{1}$ Departamento de Nutrición, Facultad de Farmacia, Universidad Complutense de Madrid, E-28040 Madrid, Spain \\ ${ }^{2}$ Unilever Foods España, División Alimentación, Spain
}

\begin{abstract}
Hypercholesterolaemia is one of the main factors contributing to the appearance and progression of CVD, which is the main cause of death in the adult population of industrialized societies. By 2020, projections suggest that it will continue to hold first place, by then causing $37 \%$ of all deaths. Therapeutic life-style changes to reduce cardiovascular risk include dietary modifications, such as the inclusion of phytosterols or plant sterols (known since the 1950s to reduce cholesterol levels). These help prevent the absorption of cholesterol and thus condition a reduction in total cholesterol and LDL-cholesterol levels, and ultimately in cardiovascular mortality. The fat-soluble nature of these sterols rendered margarine one of the best vehicles by which to supply them in the diet. Indeed, margarine was the first food to contain cholesterol-reducing phytosterols to be approved by the EU (in agreement with its regulations on new foods and food ingredients, 258/97/CE). Presently, phytosterols can be emulsified with lecithin and thus delivered in non-fat or low-fat foods and beverages. Margarine and dairy products (yoghurt and milk) enriched in phytosterols have proved better at lowering total cholesterol and LDL-cholesterol levels than have enriched cereals and their derivatives, although all can be of help, depending on the characteristics of each subject. The reduction in carotenoid bioavailability caused by sterols is minimized by increasing fruit and vegetable consumption. Individuals who habitually consume phytosterols should also follow traditional advice such as eating less dietary fat and increasing their physical activity. Phytosterols have been shown to be safe and effective in lowering cholesterol levels in many rigorous studies. In few areas of nutrition is there such consensus. Diet professionals should feel comfortable in prescribing phytosterols/stanols for the treatment of hypercholesterolaemia. They are safe whether taken alone or in combination with cholesterol-reducing drugs, such as statins and fibrates. Reinforcement counselling is essential, as therapy is effective only if compliance is good.
\end{abstract}

Plant sterols: Cholesterol: LDL-cholesterol: Cardiovascular disease

CVD is a leading cause of death in industrialized societies. Given that one of its most important risk factors is hypercholesterolaemia (Thomsen et al. 2004), effective dietary therapy aimed at reducing this problem should be a priority goal. This applies to both high- and low-risk populations (Perisee, 2005).

Therapeutic life-style changes to reduce cardiovascular risk include dietary modifications such as the inclusion of phytosterols, which effectively lower total cholesterol and LDL-cholesterol concentrations. The lifelong consumption of phytosterol esters is associated with a predicted $20 \%$ reduction in coronary events (Miettinen \& Gylling, 2004).

The purpose of the present study is to analyse the most recent results on the role of phytosterols in the control of cholesterol levels and the reduction of cardiovascular risk, differentiating between the food types used to supply these molecules.

\section{Sterols: definition and function}

Sterols are essential components of cell membranes. Cholesterol is an exclusively animal sterol, while phytosterols, produced by plants, are mainly found in seed oil (sunflower oil, maize oil, etc.), fruits, vegetables, legumes, cereals and some nuts. Over forty phytosterols have been identified, although $\beta$-sitosterol (in particular), campesterol and stigmas- terol are the most abundant and the most efficient at reducing cholesterol levels. These three sterols are structurally similar to cholesterol; all are 4-desmethyl sterols lacking methyl groups on carbon atom 4. Stanols, saturated molecules produced by the hydrogenation of sterols, are less abundant in nature (Law, 2000; Perisee, 2005). The term 'sterol' is sometimes used as a generic term to include unsaturated sterols and saturated stanols (Law, 2000).

An average intake of $200-450 \mathrm{mg}$ phytosterols is usually consumed in the diet each day (Perisee, 2005), but diets based on high intakes of vegetable fats, whole grains, fruits and vegetables have higher contents (Law, 2000). In a mixed experimental diet, concentrations of naturally occurring phytosterols were found to be inversely correlated with cholesterol absorption (Law, 2000; Ostlund, 2004). With high intakes (up to $2 \mathrm{~g}$ sterol/stanol-enriched foods daily), the intestinal absorption of cholesterol is reduced, lowering serum total and LDL-cholesterol by approximately 10-15\% in 3 weeks. This usually occurs without influencing the concentrations of HDL-cholesterol or triacylglycerols, and is independent of baseline cholesterol levels or background diet in adult hypercholesterolaemic men and women (Law, 2000; Amundsen et al. 2004; Ostlund, 2004; Perisee, 2005). On this basis, the National Cholesterol Education Program and the American Heart Association recommended the 
addition of $2 \mathrm{~g}$ phytosterols to the daily diet of adults (US Department of Health and Human Services, 2001). It is now recognized by the US Food and Drug Administration that adding sterols or stanols to the daily diet can reduce blood cholesterol levels and thereby lower the risk of CVD (Food and Drug Administration, 2000; Amundsen et al. 2004; Thomsen et al. 2004).

\section{Studies undertaken}

The cholesterol-reducing action of phytosterols has been known since the 1950s. The majority of studies report that the regular consumption of foods containing phytosterols reduces total cholesterol and LDL-cholesterol levels without modifying triacylglcerol and HDL-cholesterol concentrations (Table 1; Cleghorn et al. 2003; Amundsen et al. 2004; Clifton et al. 2004; Patch et al. 2005; Thomsen et al. 2004; Varady et al. 2004). Although some authors report HDLcholesterol levels to fall at the end of the follow-up period, the total cholesterol: HDL-cholesterol ratio is still improved (Amundsen et al. 2004).

Compliance with dietary modification may explain, to some extent, the variable cholesterol-lowering effect of phytosterols reported in studies of free-living individuals (Cleghorn et al. 2003; Patch et al. 2005; Perisee, 2005). In addition, it has been discovered that those people most likely to respond to phytosterol 'treatment' show high cholesterol absorption and low cholesterol biosynthesis rates (Ostlund, 2004).

\section{Most efficient doses}

Although studies have shown that $2 \mathrm{~g}$ phytosterols daily is the optimal therapeutic dose (Law, 2000; Ostlund, 2004; Patch et al. 2005), the response curve is linear, suggesting that an intake of around $1.5 \mathrm{~g} / \mathrm{d}$ is still clinically useful and that the levels achieved could cause a $10 \%$ change in total serum cholesterol and LDL-cholesterol levels (Law, 2000; US Department of Health and Human Services, 2001; Patch et al. 2005).

The reduction in the concentration of LDL-cholesterol at each dose is significantly greater in older than in younger people. At doses of $2 \mathrm{~g} / \mathrm{d}$, the average reduction in serum LDL-cholesterol was $0.54 \mathrm{mmol} / \mathrm{l}(14 \%)$ for participants aged $50-59$ years, $0.43 \mathrm{mmol} / 1(9 \%)$ in participants aged 40-49 years, and $0.33 \mathrm{mmol} / \mathrm{l}(11 \%)$ for those aged $30-39$ years $(P=0.005 ;$ Law, 2000). A corresponding reduction in the risk of heart disease of about $25 \%$ would be expected for this reduction in LDL-cholesterol; this is larger than the effect that would be expected by reducing the intake of saturated fat (Law, 2000).

\section{Sources of phytosterols}

Because of the solubility properties of phytosterols and stanols, they have been added (typically as esters) to oily food vehicles, such as margarines or dressings (Law, 2000; Thomsen et al. 2004). An intake of 20-25 g margarine per day is required to obtain about $2 \mathrm{~g}$ phytosterols - the optimal dose for lowering total and LDL-cholesterol (Law, 2000).

However, phytosterols can also be emulsified with lecithin and delivered in non-fat or low-fat foods and beverages
(Ostlund, 2004). This has led to the use of new food vehicles for phytosterols (Clifton et al. 2004; Thomsen et al. 2004). Clifton et al. (2004) measured the relative effects of four phytosterol ester-enriched low-fat foods (bread, breakfast cereal, milk and yoghurt) on serum lipids, plasma phytosterols and carotenoids, and reported differences depending on the food vehicle. The reduction in serum total and LDL-cholesterol levels was 8.7 and $15.9 \%$ when milk was used, and 5.6 and $8.6 \%$ when yoghurt was used. The reductions in LDL-cholesterol achieved with bread and cereals were significantly less than with phytosterol-enriched milk $(P<0 \cdot 001)$. In low-fat milk, phytosterols were almost three times more effective than in bread and cereals (Clifton et al. 2004).

These convincing results are encouraging further development of novel cholesterol-lowering, low-fat dairy products with non-esterified, non-hydrogenated phytosterols, thus expanding the food product alternatives for consumers, the functional food industry and health services (Thomsen et al. 2004).

\section{Phytosterols can be used alone or in combination with statins}

Individuals with very high concentrations of plasma cholesterol are commonly prescribed cholesterol-lowering medication, whereas those with mild-to-moderate elevations in concentration are advised, in the first instance, to make dietary changes. The advent of commercially available foods with phytosterols has the potential to offer people with moderately raised levels an additional cholesterol-lowering strategy (Cleghorn et al. 2003).

Doses of about $2 \mathrm{~g}$ plant stanols daily provided as a fatty acid ester allow about one-third of mildly hypercholesterolaemic subjects to reach acceptable cholesterol levels. Small doses of statins could be administered to individuals for whom the benefits of such monotherapy are less obvious (Miettinen \& Gylling, 2004).

Dietary combinations may not differ in potency from the effect of first-generation statins in achieving current primary prevention lipid goals. In fact, Jenkins et al. (2005) reported a similar reduction in LDL-cholesterol in thirty-four hyperlipidaemic subjects who followed a low-saturated fat diet and who were administered either $20 \mathrm{mg}$ lovastatin or phytosterols at $1.0 \mathrm{~g} / 1000 \mathrm{kcal}$.

The addition of phytosterol/stanol to foods increases their cost, but they are still cheaper than daily statins (Perisee, 2005).

\section{Safety and risks}

The goal is to achieve successful lipid reduction without adverse side-effects or unacceptable body/dietary profile changes. Currently, the evidence suggests that phytosterol/ stanol use is safe, but long-term results ( $>5$ years) are lacking. Whether statin doses can be safely and cost-effectively reduced by the addition of phytosterol/stanol products is yet to be determined (Perisee, 2005).

A few studies have suggested that a slight reduction in the plasma concentration of some carotenoids occurs with consumption of phytosterol-enriched foods (Clifton et al. 2004), but this finding has not been consistent (Amundsen et al. 2004), particularly when lipid-adjusted concentrations are 
Phytosterols and cardiovascular health

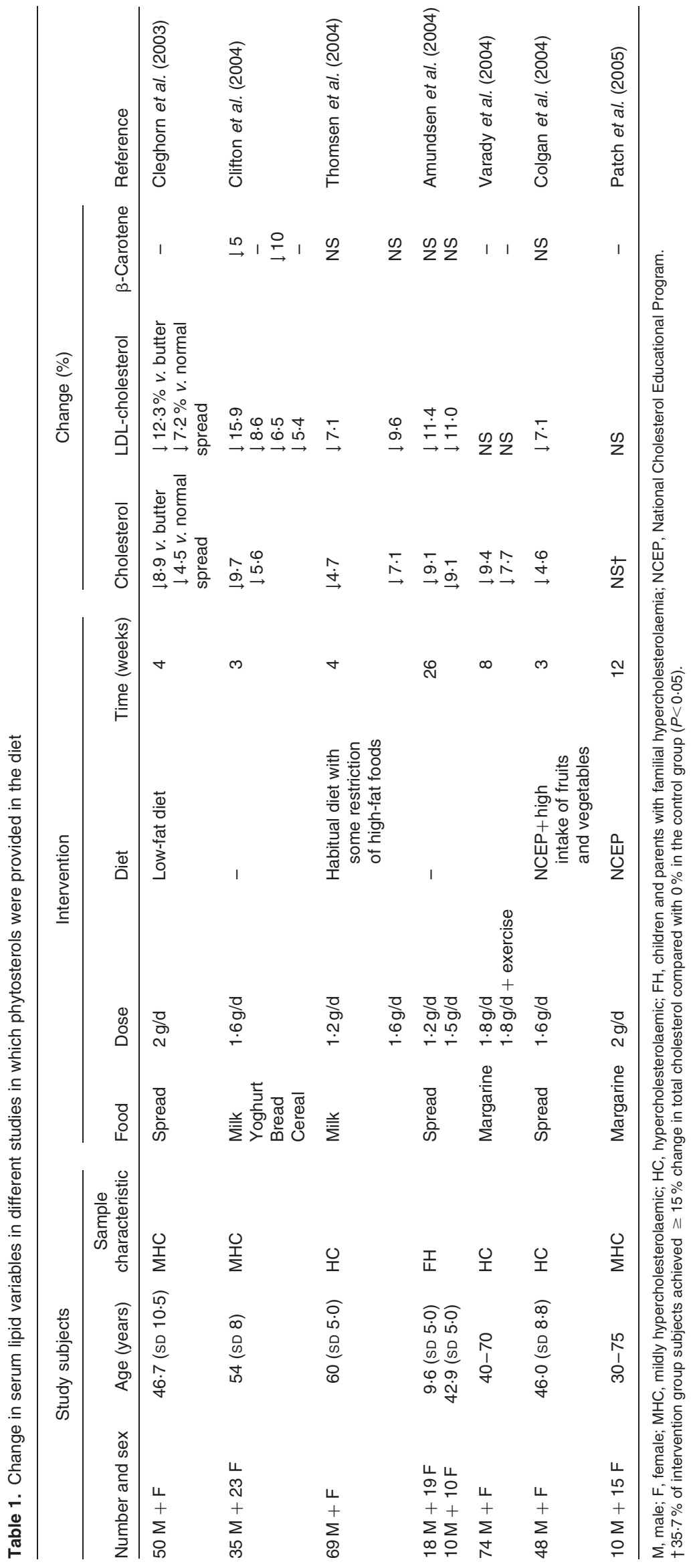


compared (Thomsen et al. 2004; Perisee, 2005). Indeed, it would seem that provided the background diet is appropriate and includes a variety of carotenoid-rich foods, any alteration in plasma carotenoids is likely to be minor and of little or no clinical significance (Cleghorn et al. 2003; Amundsen et al. 2004).

It is recommended by the National Cholesterol Education Program that five servings of vegetables and fruits be consumed in the daily diet. This has been shown to effectively maintain plasma levels of antioxidants when sterol or stanol supplements are taken (Noakes et al. 2002; Perisee, 2005). In the latest National Cholesterol Education Program guidelines, the substantial advantage of these innovative dietary approaches is rigorously emphasized (Perisee, 2005).

\section{Should other dietary modifications be made?}

Apart from the consumption of phytosterols, increasing the amount of fruit and vegetables consumed within a balanced and sufficient diet would seem to reduce cardiovascular risk. The same is true of regular exercise. In comparison with phytosterols or exercise alone, their combination leads to more beneficial changes in lipid profiles. The implementation of such combination therapy might improve lipid profiles in those at risk of coronary artery disease (Varady et al. 2004).

In the effective management of hyperlipidaemia, there are a number of dietary approaches that can achieve moderate results. These include reducing the amount of saturated fat in the diet, increasing the intake of $n-3$ PUFA fats, and increasing the intake of dietary fibre. It is important that the introduction of novel dietary strategies does not adversely affect the dietary profile (Patch et al. 2005).

In people with moderately raised plasma cholesterol consuming reduced-fat diets, the reduction in plasma total and LDL-cholesterol concentrations achieved by replacing butter with a polyunsaturated spread is enhanced by the addition of phytosterols (Cleghorn et al. 2003).

Phytosterols/stanols have additive effects with other dietary modifications (Law, 2000) that should not be disregarded (Patch et al. 2005).

\section{Limits and final discussion}

It is not a good idea to transmit to the population that the consumption of foods containing phytosterols is the solution to all problems. Teaching the characteristics of a balanced, varied and sufficient diet should still be the priority. However, within this context, foods containing phytosterols offer an interesting weapon in the fight against CVD. Such an approach has the advantage that it is not restrictive, which should help avoid deficiencies.

Many rigorous investigations have shown that an intake of $2 \mathrm{~g}$ phytosterols (considered the optimum daily dose) helps regulate cholesterolaemia, LDL-cholesterol levels and the risk of CVD. In few areas of nutrition is there such consensus. Since phytosterols/stanols reduce serum cholesterol levels but might also reduce plasma $\beta$-carotene levels (which can be compensated by an adequate intake of fruits and vegetables), and given that there is no evidence that intakes of $>3 \mathrm{~g} / \mathrm{d}$ provide any additional benefits in terms of cholesterol reduction, it seems prudent to mention this on the labels of phytosterol-containing foods (in accordance with EU regulation 608/2004/CE, 31 March 2004, concerning foods and food ingredients with added sterols/stanols and their esters).

\section{Role of health professionals}

Strategies to lower plasma cholesterol concentrations are of great public health importance since raised concentrations are a major risk factor for CHD. Further, clinical interventions have shown that reducing total and LDL-cholesterol levels significantly reduces CHD mortality (Cleghorn et al. 2003).

As indicated by Patch et al. (2005), dietary compliance is an essential component of measuring the effectiveness of therapy. Diet professionals should play a major role in reinforcing the importance of adhering to therapy stipulations (Perisee, 2005). The routine prescription of foods containing phytosterols/stanols could be an effective strategy in the management of hypercholesterolaemic patients in the clinical setting (Patch et al. 2005).

Finally, future research needs to be directed towards determining the potential costs and benefits of increasing the variety of foods fortified with phytosterols/stanols (Perisee, 2005).

\section{References}

Amundsen ÅL, Ntanios F, van der Put N \& Ose L (2004) Long-term compliance and changes in plasma lipids, plant sterols and carotenoids in children and parents with FH consuming plant sterol esterenriched spread. Eur J Clin Nutr 58, 1612-1620.

Cleghorn CL, Skeaff CM, Mann J \& Chisholm A (2003) Plant sterolenriched spread enhances the cholesterol-lowering potential of a fat-reduced diet. Eur J Clin Nutr 57, 170-176.

Clifton PM, Noakes M, Sullivan D, et al. (2004) Cholesterol-lowering effects of plant sterol esters differ in milk, yoghurt, bread and cereal. Eur J Clin Nutr 58, 503-509.

Colgan HA, Floyd S, Noone EJ, Gibney MJ \& Roche HM (2004) Increased intake of fruit and vegetables and low-fat diet, with and without low-fat plant sterol-enriched spread consumption: effects on plasma lipoprotein and carotenoid metabolism. J Hum Nutr Diet 17, 561-569.

Food and Drug Administration (2000) Food labeling: health claims; plant sterol/stanol esters and coronary heart disease. Food Drug Adm Fed Reg 65, 54686-54739.

Jenkins DJA, Kendall CWC, Marchie A, et al. (2005) Direct comparison of a dietary portfolio of cholesterol-lowering foods with a statin in hypercholesterolemic participants. Am J Clin Nutr 81, $380-387$.

Law M (2000) Plant sterol and stanol margarines and health. BMJ 320, $861-864$

Miettinen TA \& Gylling H (2004) Plant stanol and sterol esters in prevention of cardiovascular diseases. Ann Med 36, 126-134.

Noakes M, Clifton P, Ntanios F, Shrapnel W, Record I \& McInerney J (2002) An increase in dietary carotenoids when consuming plant sterols or stanols is effective in maintaining plasma carotenoid concentrations. Am J Clin Nutr 75, 79-86.

Ostlund RE Jr (2004) Phytosterols and cholesterol metabolism. Curr Opin Lipidol 15, 37-41.

Patch CS, Tapsell LC \& Williams PG (2005) Plant sterol/stanol prescription is an effective treatment strategy for managing 
hypercholesterolemia in outpatient clinical practice. $J$ Am Diet Assoc 105, 46-52.

Perisee DM (2005) Food fortification with plant sterol/stanol for hyperlipidemia: management in free-living populations. $J \mathrm{Am}$ Diet Assoc 105, 52-53.

Thomsen AB, Hansen HB, Christiansen C, Green $\mathrm{H}$ \& Berger A (2004) Effect of free plant sterols in low-fat milk on serum lipid profile in hypercholesterolemic subjects. Eur J Clin Nutr 58, $860-870$.
US Department of Health and Human Services (2001) Expert Panel on Detection, Evaluation, and Treatment of High Blood Cholesterol in Adults (Adult Treatment Panel III): executive summary of the third report of the National Cholesterol Education Program (NCEP). JAMA 28, 2486-2497.

Varady KA, Ebine N, Vanstone CA, Parsons WE \& Jones PJ (2004) Plant sterols and endurance training combine to favorably alter plasma lipid profiles in previously sedentary hypercholesterolemic adults after 8 wk. Am J Clin Nutr 80, 1159-1166. 